\title{
Super-Atmospheric Pressure Electrospray Ion Source: Applied to Aqueous Solution
}

\author{
Lee Chuin Chen, ${ }^{1}$ Mridul Kanti Mandal, ${ }^{2}$ Kenzo Hiraoka ${ }^{2}$ \\ ${ }^{1}$ Interdisciplinary Graduate School of Medicine and Engineering, University of Yamanashi, 4-3-11 Takeda, Kofu, Yamanashi, \\ 400-8511, Japan \\ ${ }^{2}$ Clean Energy Research Center, University of Yamanashi, 4-3-11 Takeda, Kofu, Yamanashi, 400-8511, Japan
}

\begin{abstract}
This is a follow-up paper of our previous report on an ion source, which was operated at an operating pressure higher than the atmospheric pressure. Besides having more working gas for desolvation, the reduction of mean free path of electrons in a higher pressure environment increases the threshold voltage for gaseous breakdown, thus enabling a stable electrospray for the sample solution with high surface tension without the occurrence of electric discharge. In our previous work, the ion source was not coupled directly to the mass spectrometer and significant amount of ions were lost before entering the vacuum of the mass spectrometer. In this paper, we report the new design of our second prototype in which, by using a modified ion transport capillary, the pressurized ESI ion source was coupled directly to the first pumping stage of the mass spectrometer without additional modification on the vacuum pumping system. Demonstrations of the new ion source on the sensitive detection of native proteins from aqueous solution in both positive and negative ion modes are presented.
\end{abstract}

Key words: High pressure, Super-atmospheric pressure, ESI-MS, Aqueous solution, Corona discharge, Native protein

\section{Introduction}

S tandard electrospray ion source is operated under an atmospheric pressure ambient [1-4]. Working at atmospheric pressure is not only convenient to the user, the bath gas also provide sufficient thermal energy to the charged droplets for the vaporization of solvent without freezing them. This feature allows the electrospray to work with wide range of solvents including those commonly used in the liquid chromatography, while its vacuum counterpart electrohydrodynamic (EHD) spray [5, 6] can only handle limited types of solvent. Generation of ions in the atmospheric pressure rather than in vacuum

Electronic supplementary material The online version of this article (doi:10.1007/s13361-011-0253-2) contains supplementary material, which is available to authorized users.

Correspondence to: Lee Chuin Chen; e-mail: leechuin.yamanashi@gmail.com and Kenzo Hiraoka; e-mail: hiraoka@yamanashi.ac.jp also helps to keep the internal and translational energies of the ions at a level corresponding to the bath gas temperature [3]. Nowadays, the mass spectrometer, which is designed to work with ESI and other atmospheric pressure ionization (API) source, is usually referred as API mass spectrometer [7].

Some efforts had been put forward to explore the workability of ESI in the pressure range other than atmospheric pressure. For example, sub-atmospheric pressure electrospray (few 10s Torr) has been attempted by Marginean and coworkers using ESI emitter with solution flow rate of $\mathrm{nL} / \mathrm{min}$, so that the generated ions could be guided more efficiently by their focusing ion optics, namely ion funnel to the mass analyzer located in high vacuum $[8,9]$. On the other hand, we have recently demonstrated the super-atmospheric pressure electrospray ionization with an ion source that could be pressurized with compressed air up to 5 bars [10]. Raising the operating pressure of the ESI ion source offers some 
interesting features that can supplement the conventional ESI or nanoESI ion source, especially in the case of handling labile compound in aqueous solution. Aqueous solution has so far been handled by pneumatic-assisted ESI [11, 12], heated ESI [13], ESI with electron scavenging sheath gas [14], and more popularly by nanoESI [15-17]. The approach used in our ion source is somewhat different from these existing methods.

Due to its high surface tension, water is known to have higher ESI onset voltage compared with organic solvent mixtures (e.g., $50 \% \mathrm{vol} / \mathrm{vol}$ methanol/water solution). Thus, even with pneumatic assistance, it is rather difficult to achieve stable and efficient electrospray ionization for aqueous solution because the threshold voltage for the gaseous breakdown could fall below or about the same with the onset voltage of electrospray. Once the gaseous breakdown occurs, it leads to a corona or arc discharge that seriously affects the electric field near the ESI emitter and degrades the performance of electrospray. Theoretical estimations for the onset voltage of electrospray are given by Taylor [18], Smith [19], and more recently by Wilm and Mann [15]. The ESI onset voltage depends primarily on the surface tension of the solution and the relative permittivity of the ambient medium. If the ambient medium is air or gas, and for a solution which is relatively incompressible (e.g., water), the electrospray onset voltage is almost unaffected by the ambient gas pressure up to several bars [10]. The threshold voltage for inducing the gaseous breakdown however, will increase with the rise of gas pressure due to the reduction of mean free path for electrons. This phenomenon is usually referred as Paschen law [20]. Thus, besides using $\mathrm{CO}_{2}$ or $\mathrm{SF}_{6}$ as sheath gas [14, 21], it is also possible to quench the gaseous breakdown completely by pressurizing the ESI ion source with air or $\mathrm{N}_{2}$ to an appropriate level.

By operating the ion source at air pressure higher than 4 bars, we have previously verified that asides from maintaining stable steady cone-jet mode, electric field around the ESI emitter could be increased up to the formation of multi-jet mode (nomenclature due to Cloupeau and Prunet-Foch [22]) for both positive and negative ion modes without the occurrence of corona discharge. Besides solving the gaseous breakdown problem, there are other advantages by working under a high pressure condition. Since more bath gas is used for the desolvation process, the electrospray ionization is expected to be even softer compared to that operated at atmospheric pressure. The gas pressure of the ion source can also be adjusted to tune the pressure in the first pumping stage of the mass spectrometer which is known to influence the collision induced dissociation and the softness of ion desolvation in vacuum [23-25]. When the heating of the sample solution becomes necessary, (e.g., to study the thermally induced denaturation of protein, DNA etc.), a high pressure condition can even allow the solution to be heated beyond its boiling point to realize a super-heated electrospray ionization.
In this paper, we report a new design of our second prototype of the high pressure ESI source. In our previous prototype, ions produced from the ESI were not coupled directly to the vacuum stage of the mass spectrometer, but were transported out from the high pressure vessel to an atmospheric pressure side via a metallic capillary. The inner diameter (i.d.) of the capillary was $0.8 \mathrm{~mm}$ and the gas flow rate was $\sim 17 \mathrm{~L} / \mathrm{min}$. The typical gas sampling rate for API mass spectrometers with differential pumping and $\sim 0.5 \mathrm{~mm}$ inlet orifice is about $1 \mathrm{~L} / \mathrm{min}$. This means that most of the ion rich gas from our previous ion source could not be fed directly to the mass spectrometer due to the limitation of gas load allowed for maintaining the operational vacuum level. In the present work, endeavor has been made to transport the electrospray ions and fine charged droplets directly from the high pressure vessel to the vacuum stage of the mass spectrometers.

\section{Experiment}

\section{Mass Spectrometers}

The functionality of the present ion source has been tested using two commercial mass spectrometers: a linear ion trap (LTQ-Velos; Thermo Fisher Scientific, San Jose, CA, USA), and a bench top Orbitrap (Exactive; Thermo Fisher Scientific, Bremen, Germany). Working pressures in the highest vacuum stages that house the mass analyzer were $10^{-5}$ mbar for LTQVelos, and $10^{-10}$ mbar for Exactive.

LTQ-Velos is a recently released linear ion trap from Thermo Fisher Scientific in which the length of the ion transport capillary $(\sim 6 \mathrm{~cm})$ is nearly half of that used in the previous version, and a focusing stacked ring ion guide is employed instead of a standard inlet-skimmer arrangement. This focusing ion guide is somewhat different from Smith's ion funnel in such a way that ring electrodes with same i.d. are progressively spaced rather than with progressively reduced i.d. [26]. The Exactive utilizes a typical nozzleskimmer arrangement [7, 27], and a $\sim 10 \mathrm{~cm}$ long heated ion transport capillary is used to transport ions from the atmospheric pressure ambient to the first vacuum stage. Both instruments utilize a simple ball valve that allows the ion transport capillary to be easily removed for cleaning and re-installed without affecting the vacuum of the mass analyzer.

The original ion transport capillaries for the two mass spectrometers have an i.d. of $0.55 \mathrm{~mm}$ and the pressure in the first pumping stage is $1.7 \sim 2$ mbar. To couple the high pressure ion source directly to the mass spectrometer while maintaining the normal operating pressure in the first pumping stage, ion transport capillaries with $0.25 \mathrm{~mm}$ in i.d., and $1.59 \mathrm{~mm}$ (1/16 inch) in outer diameter (o.d.) were fabricated and employed throughout the experiment. These custom made ion transport capillaries were made from commercial straight cut stainless steel capillaries (GL Sciences, Tokyo, Japan). 
Photographs of these ion transport capillaries are shown in Supplementary Figure S1 of the supporting information. No additional modification was made on the vacuum pumping system.

\section{Super-Atmospheric Pressure ESI Source}

Simplified schematic of the high pressure ESI (HP-ESI) ion source is depicted in Figure 1 and photographs showing the experimental setup of the ion source are shown in Supplementary Figure S2. The body of the desolvation chamber was made of aluminum alloy, which was bored to an i.d. of $60 \mathrm{~mm}$. The desolvation chamber was attached with two view ports made of transparent polycarbonate polymer. The electrospraying condition could be inspected using a long working distance microscope via these view ports. The ion source chamber was pressurized with dry air supplied from an air compressor (oil-free scroll compressor; Anest Iwata, Yokohama, Japan). The pressure of the ion source was monitored by an analog pressure gauge and was adjusted by a speed valve and a pressure regulator equipped with the air compressor. On one side of the desolvation chamber, a through hole with diameter of $1.6 \mathrm{~mm}$ was bored so that the ion transport capillary (with o.d. of $1.59 \mathrm{~mm}$ ) could be inserted into the desolvation chamber with a narrow air gap in between them.

ESI emitter was a stainless steel capillary with i.d. of $50 \mu \mathrm{m}$ (New Objective, Woburn, MA, USA). Distance between the ESI emitter tip and the ion transport capillary (counter electrode) was $6 \sim 10 \mathrm{~mm}$ (adjustable during the experiment). Sample solution was pumped through the ESI capillary using a syringe pump with high linear force (PHD 4400; Harvard Apparatus, MA, USA). The flow rates of sample solution were $0.4 \sim 5 \mu \mathrm{L} / \mathrm{min}$. Under typical operation, the ion source was pressurized to $6 \sim 7$ bars and the flow rate of air supplied to the desolvation chamber was about $15 \sim 20 \mathrm{~L} / \mathrm{min}$. The ion transport capillary protruded into the ion source chamber and sampled the ion rich gas at a flow rate of $\sim 1 \mathrm{~L} / \mathrm{min}$. Nearly all the working gas was exhausted out from the ion source via the air gap between the ion transport capillary (o.d. $=1.59 \mathrm{~mm}$ ) and the through hole of $1.6 \mathrm{~mm}$ in diameter, carrying out the moisture from the desolvation chamber. Although the flow rate of the flowing air was large $(15 \sim 20 \mathrm{~L} / \mathrm{min})$, the wind speed around the ESI emitter was low (in the order of $\sim 0.1 \mathrm{~m} / \mathrm{s}$ ), because of the large i.d. of the ion source chamber. Compared with those of ion spray [11], sonic spray [28], and electro-sonic spray [12] with high speed nebulizing gas, the spraying of liquid in the present ion source was driven electrostatically like an unassisted electrospray.

\section{Sample Preparation}

Bovine insulin and myoglobin and cytochrome $c$ were purchased (Sigma, St. Louis, MO, USA) and were used without further purification. The primary stock solution of bovine insulin was prepared in $0.1 \%$ trifluoroacetic acid (TFA) in aqueous solution, and was subsequently diluted with pure water to $10^{-6}$ and $10^{-9} \mathrm{M}$. Myoglobin and cytochrome $c$ were

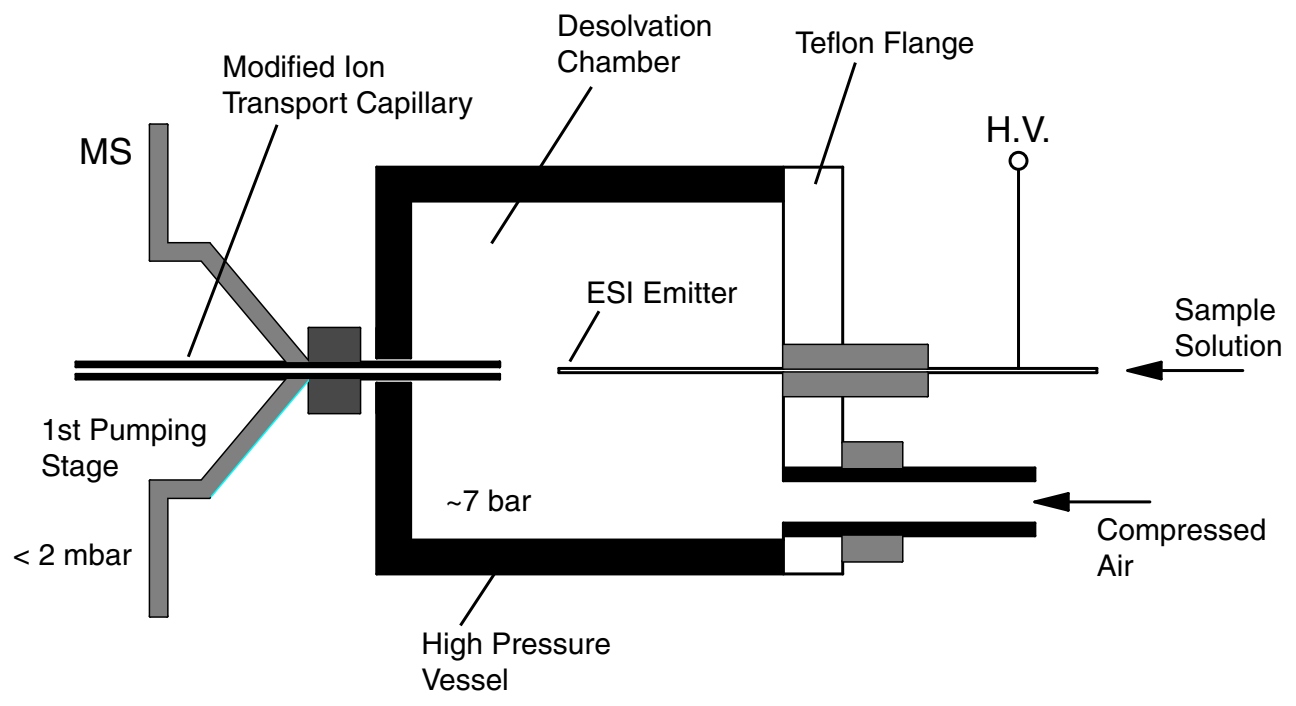

Figure 1. Simplified schematic of the high pressure ESI ion source. The ESI ion source was pressurized with dry air to $\sim 7$ bars and the vacuum pressure in the first pumping stage of the mass spectrometer was maintained at below 2 mbar, using a modified ion transport capillary with i.d. of $0.25 \mathrm{~mm}$, and o.d. of $1.59 \mathrm{~mm}(1 / 16 \mathrm{in})$. On one side of the desolvation chamber, a through hole with diameter of $1.6 \mathrm{~mm}$ was bored so that the ion transport capillary could be inserted into the desolvation chamber with a narrow air gap between them. Distance between the ESI emitter tip and the ion transport capillary was $6 \sim 10 \mathrm{~mm}$. Sample solution was pumped through the ESI emitter capillary using a syringe pump with high linear force. 1 bar $\approx 1$ atm 
dissolved in pure water and $10 \mathrm{mM}$ ammonium acetates aqueous solution. Pure water was prepared using Milli-Q system (Millipore, Bedford, MA).

\section{Results and Discussion}

Relationships between the pressure in the first pumping stage of the mass spectrometers, $P_{1}$, and the operating pressures for the HP-ESI ion source, $P_{0}$, are depicted in Figure 2a for LTQ-Velos and Exactive. Pressures in the highest vacuum stages for both instruments (LTQ-Velos \& Exactive) were found to be unaffected by the ion source pressure under the tested pressure range. Figure $2 \mathrm{~b}$ is the plot of gas sampling rate, $Q_{\mathrm{s}}$, (which is the flow rate of air sampled through the ion transport capillary into the first vacuum stage of the mass spectrometers) versus the ion source pressure. The gas sampling rate was measured by connecting a precision mass flow meter and pressure gauge to the inlet of the ion transport capillary under a vacuum sealed condition.
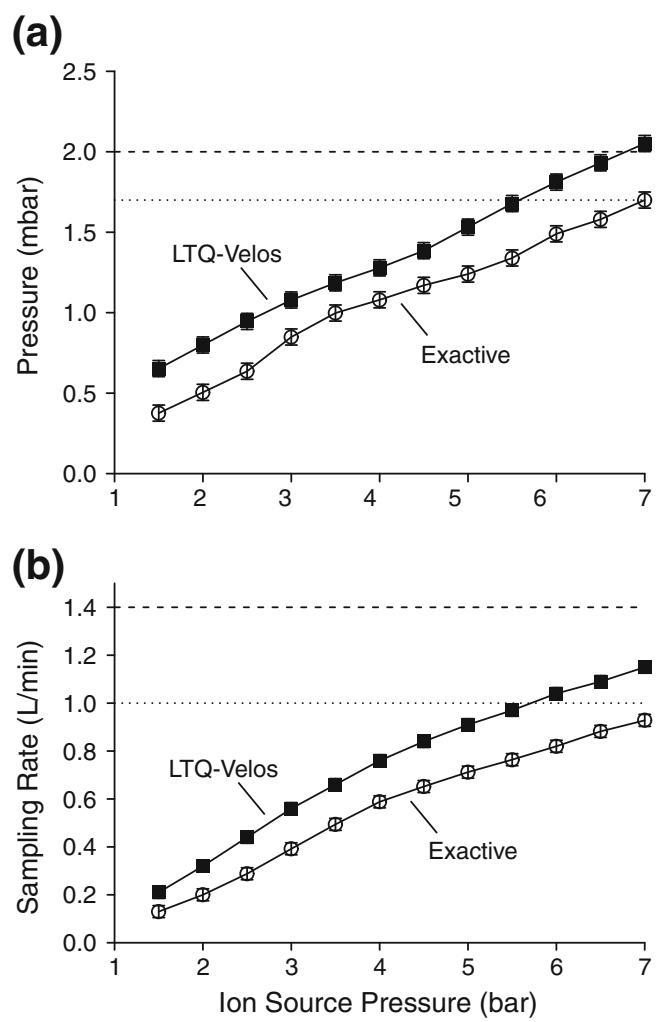

Figure 2. Plots of (a) pressure in the first pumping stage $P_{1}$, and (b) gas sampling rate, $Q_{\mathrm{S}}$ of the mass spectrometer versus the operating pressures of the ion source. Solid square: LTQVelos; open circle: Exactive. Temperature of the ion transport capillary was $300^{\circ} \mathrm{C}$. Original working conditions for $P_{1}$, and $Q_{\mathrm{S}}$ were: $2 \mathrm{mbar}$ and $1.4 \mathrm{~L} / \mathrm{min}$ for LTQ-Velos (dashed lines), and $1.7 \mathrm{mbar}$ and $1 \mathrm{~L} / \mathrm{min}$ (dotted lines) for Exactive, respectively. Pressures in the highest vacuum stages were $1.76 \times 10^{-5}$ mbar for LTQ-Velos and $1.12 \times 10^{-10}$ mbar for Exactive, and these value were not affected by the change of ion source pressure
Original working conditions for $P_{1}$ and $Q_{\mathrm{S}}$ were: 2 mbar and $1.4 \mathrm{~L} / \mathrm{min}$ for LTQ-Velos and $1.7 \mathrm{mbar}$ and $1 \mathrm{~L} / \mathrm{min}$ for Exactive. For an API mass spectrometer, $P_{1}$ is an important parameter because it determines the location of the mach disk of the free jet expansion in the vacuum [4], affects the desolvation of analyte-solvent clusters [24], and influences the focusing condition of the ion guide located in the subsequent vacuum stage. As shown in Figure 2, as the pressure of the ion sources was increased to $6 \sim 7$ bars, both $P_{1}$ and $Q_{\mathrm{S}}$ approach to the original working condition. As for the distance of Mach disk from the source of the jet, $X_{\mathrm{M}}$, it can be roughly estimated by $X_{\mathrm{M}}=(2 / 3) D\left[P_{0} / P_{1}\right]^{1 / 2}$, where $D$ is the diameter of the ion transport capillary, $P_{0}$ is the upstream pressure (high pressure side), and $P_{1}$ is the downstream pressure in the first vacuum chamber [4]. If $P_{\mathrm{o}}=7$ bars (which was the typical operating condition in this work), deviations of $X_{\mathrm{M}}$ from their original designed values were about $20 \%$ for both Exactive and LTQ-Velos.

Figure $3 \mathrm{a}$ and $\mathrm{b}$ show the plot of ion intensities versus the ion source pressure for Exactive and LTQ-Velos in the positive ion mode. Two sets of sample were tested here. One was $10^{-6} \mathrm{M}$ bovine insulin in $50 \% \mathrm{vol} / \mathrm{vol}$ methanol/water solution, which is a solvent commonly used for atmospheric pressure ESI, and another one was $10^{-6} \mathrm{M}$ bovine insulin in pure aqueous solution. The solution flow rate was $2 \mu \mathrm{L} / \mathrm{min}$ for both samples, and the temperature of the heated ion transport capillaries was kept at $300{ }^{\circ} \mathrm{C}$. The ESI voltages were individually optimized at 4.5 and $6 \mathrm{kV}$, respectively, for methanolic and aqueous solutions. Major peaks originated from insulin have the charge states of $+3,+4$, and +5 , and the intensities depicted in Figure 3 are the summed value of these peaks. Basically, the ion intensities increase with the ion source pressure and reach a plateau at about 6 bars for Exactive (Figure 3a), and 4 bars for LTQ-Velos (Figure $3 b$ ). It is also seen in Figure 3 that when the ion source pressure was optimized, the abundance of ions produced from aqueous solution could achieve the same order of magnitude as that of organic solvent mixture. When the ion source pressure was reduced to lower than 2.5 bars, ion intensities drop drastically for the case of aqueous solution due to the occurrence of corona discharge.

Regarding the sensitivity, it has been improved by more than an order of magnitude compared with the system in our previous report [10]. As shown in Figure 4a, the bovine insulin in aqueous solution could be easily detected down to the level of $10^{-9} \mathrm{M}$ with signal to noise ratio, $\mathrm{S} / \mathrm{N}>10$. For comparison, solution with the same concentration of sample was prepared again using $50 \% \mathrm{vol} / \mathrm{vol}$ methanol/water mixture as solvent and was analyzed under atmospheric pressure condition using the original ion transport capillary (i.d. $=0.55 \mathrm{~mm}$ ). The mass spectrum obtained by atmospheric pressure ESI is depicted in Figure 4b. Same ESI emitter was used in these measurements with the solution flow rate of $1 \mu \mathrm{L} / \mathrm{min}$. Each mass spectrum depicted here was the average of 10 single scan mass spectra with $50 \mathrm{~ms}$ injection time. 
(a)
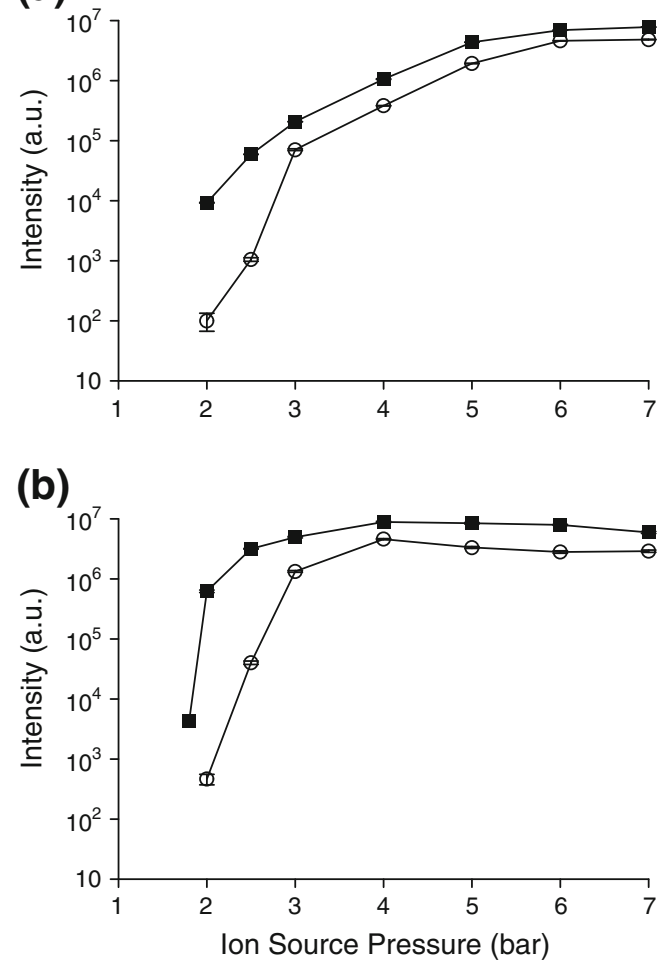

Figure 3. Plots of ion intensities for $10^{-6} \mathrm{M}$ bovine insulin in different solvents versus ion source pressures using (a) Exactive, and (b) LTQ-Velos. Solid squares are for $50 \% \mathrm{vol} /$ vol methanol/water mixture, and open circles are for $100 \%$ aqueous solution. Solution flow rate was $2 \mu \mathrm{L} / \mathrm{min}$ and the temperature of the heated ion transport capillary was $300^{\circ} \mathrm{C}$. The ion intensities depicted here are the sum of peak intensities for charge states from +3 to +5 . The voltages applied to the ESI emitter were individually optimized at 4.5 and $6 \mathrm{kV}$, respectively, for methanolic and pure aqueous solution. In the case of aqueous solution, corona discharge took place during the measurement taken at ion source pressure of 2 and 2.5 bars

Under a viscous flow regime, the conductance $C$ of a gas transport tube with i.d. $D$, and length $L$, is proportional to $P_{A} D^{4} / L$, where $P_{A}$ is the average between the upstream and downstream pressure [29]. In this experiment, $D_{\text {original }}=$ $0.55 \mathrm{~mm}, D_{\text {modified }}=0.25 \mathrm{~mm}, P_{A \text {, original }}=1 \mathrm{bar}$, and $P_{A}$, modified $=7$ bars, thus the ratio of conductance between the modified and the original ion transport capillaries, $C_{\text {modified }}$ $C_{\text {original }}=0.29$. Although the gas throughput for the HP-ESI was about the same with that of AP-ESI owing to higher operating pressure, lower ion transmission efficiency was anticipated for the modified ion transport capillary [30]. As shown in Figure 4, ion intensities for insulin measured by HP-ESI (Figures 4a) were about 2.5 times lower compared with those of AP-ESI (Figure 4b). Nevertheless, this loss factor was moderately low considering that the i.d. of our modified ion transport capillary has been reduced to less than half of the original one.
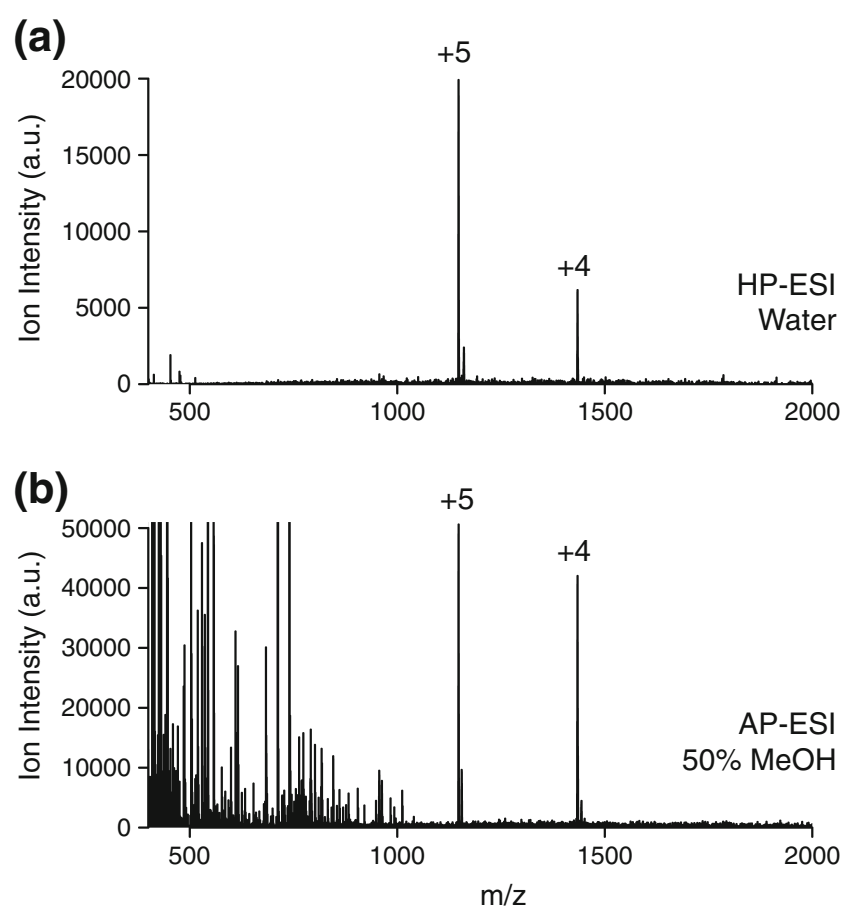

Figure 4. Mass spectra for (a) $10^{-9} \mathrm{M}$ bovine insulin in pure water acquired by high pressure ESI at 7 bars, and (b) $10^{-9} \mathrm{M}$ bovine insulin in $50 \% \mathrm{vol} / \mathrm{vol}$ methanol/water mixture acquired by atmospheric pressure ESI using the original ion transport capillary (i.d. $=0.55 \mathrm{~mm}$ ). Solution flow rate was $1 \mu \mathrm{L} / \mathrm{min}$ and the ESI emitter potentials in (a) and (b) were individually optimized at 6 and $3.6 \mathrm{kV}$, respectively. These mass spectra were taken by Exactive and each mass spectrum represents the average of 10 single scan mass spectra with 50 ms injection time

In sum, in the case of ESI friendly solvent, the high pressure ESI ion source at the present stage was not ready to outperform the atmospheric pressure ESI due to a higher ion transmission loss, and further work is necessary in the future to improve this aspect. Nevertheless, the present ion source has been proven to be useful for the sensitive MS analysis of aqueous sample solution without the occurrence of gaseous breakdown. It is also interesting to note that at low analyte concentration, the mass spectrum obtained from pure aqueous solution (Figure 4a) appeared to be much cleaner compared with those obtained from organic solvent mixture (Figure 4b), possibly owing to less contaminants present in the pure water.

Positive and negative HP-ESI mass spectra of myoglobin and cytochrome $c$, each of $10^{-7} \mathrm{M}$ in two different aqueous solutions: pure water and $10 \mathrm{mM}$ aqueous ammonium acetate are shown in Supplementary Figure S3. Myoglobin is thermally labile, and the iron-containing porphyrin (heme), which is bound by noncovalent bonds, can be easily detached if excessive energy is deposited to the protein molecules during the ionization process. Under a mild setting on the desolvation parameters (see caption in Figure S3), apomyoglobin (without heme) and heme were nearly 
absent in the mass spectra, and all the dominant peaks were originated from the holomyoglobin (with heme). In the cases of freshly prepared sample in pure water ( $\mathrm{pH} 7)$, positive mass spectra of myoglobin and cytochrome $c$ show a distribution of peaks with higher charge states, indicating some change of protein conformation from its native state, which could be due to the local $\mathrm{pH}$ change originating from the electrochemical reaction that is inherent in electrospray [31]. With the use of $10 \mathrm{mM}$ aqueous ammonium acetate, which is the most popular buffer for the electrospray of native protein, the detected peaks were shifted to a lower charge state, for example +8 in the case of myoglobin and +7 for cytochrome $c$.

Negative ESI mass spectra of these proteins show some considerable difference with the positive mode detection. First, in the case of pure water, the detected protein anions appeared at much lower charge state in contrast to the case of cations. With the use of ammonium acetate buffer, the charge state distribution became narrower like the positive ion mode, but the major peaks in the negative mass spectra still have one or two charge states lower compared to those in the positive ion mode. This was perhaps due to an unequal number of acidic and basic sites which are exposed to the outer surface of the tightly packed protein structure. However, this interpretation is still speculative and we are cautioned by some early works $[32,33]$ that the observed mass spectra may not represent the condition of protein in the solution state. Studies done by Konermann and Douglas concluded that for unfolded proteins, only positive-ion spectra provide reasonable charge state distribution that reflects the protein shapes in solution [32]. For native protein, it is not very clear yet because the apparent discrepancies in the positive and negative ESI spectra are also related to the ionization processes and gas phase stabilities of cations and anions [33]. More work on this aspect is certainly needed for a better interpretation of positive and negative ESI mass spectra, and we hope the present ion source could be a useful tool for research in this area.

\section{Conclusion}

We have demonstrated that super-atmospheric pressure electrospray ion source could be easily coupled to commercial mass spectrometers for sensitive detection of analytes in aqueous solution, in both positive and negative ion modes. The approach used in this paper allows the ion source to be easily removed or installed to the mass spectrometer in a "plug-and-play" manner like the originally equipped ESI and APCI ion sources that come along with the mass spectrometer instrument. Besides electrospray of native protein, the present ion source is also potentially useful for the gradient elution chromatography, which involves water-organic solvent solution, and the absence of corona discharge can ensure a stable and sensitive analysis over the full range of solvent composition. Aside from the field of mass spec- trometry, high pressure electrospray can also be used to deliver fine water aerosol for applications such as pulmonary drug delivery or chemical synthesis, where the use of toxic organic solvent or the use of $\mathrm{CO}_{2}$ and $\mathrm{SF}_{6}$ are not desirable. Although it was demonstrated here only with electrospray, the concept of super-atmospheric pressure ion source can also be extended to other ionization techniques, particularly for those used in the ionization of gaseous compounds because the number of gas phase interaction is increased in a higher pressure condition. Electric discharge based method such as that used in APCI may not be suitable for this purpose, but it can be implemented with photochemical or secondary electrospray ionization $[34,35]$.

\section{Acknowledgment}

The authors acknowledge support for this work by the Program to Disseminate Tenure Tracking System from the Ministry of Education, Culture, Sports, Science, and Technology of the Japanese government.

\section{References}

1. Dole, M.: Molecular beams of macroions. J. Chem. Phys. 49, 2240 (1968)

2. Yamashita, M., Fenn, J.B.: Electrospray ion source. Another variation on the free-jet theme. J. Phy. Chem. 88, 4451-4459 (1984)

3. Fenn, J.B., Mann, M., Meng, C.K., Wong, S.F., Whitehouse, C.M.: Electrospray ionization - principles and practice. Mass Spectrom. Rev. 9, 37-70 (1990)

4. Fenn, J.B.: Mass spectrometric implications of high-pressure ion sources. Int. J. Mass. Spectrom 200, 459-478 (2000)

5. Evans, C.A.: An electrohydrodynamic ion source for the mass spectrometry of liquids. Rev. Sci. Instrum. 43, 1527 (1972)

6. Simons, D.S., Colby, B.N., Evans, C.A.: Electrohydrodynamic ionization mass spectrometry - the ionization of liquid glycerol and nonvolatile organic solutes. Int. J. Mass. Spectrom. Ion Phys. 15, 291-302 (1974)

7. Covey, T.R., Thomson, B.A., Schneider, B.B.: Atmospheric pressure ion sources. Mass Spectrom. Rev. 28, 870-897 (2009)

8. Marginean, I., Page, J.S., Kelly, R.T., Tang, K., Smith, R.D.: Effect of pressure on electrospray characteristics. Appl. Phys. Lett. 95, 184103 (2009)

9. Marginean, I., Page, J.S., Tolmachev, A.V., Tang, K., Smith, R.D.: Achieving $50 \%$ ionization efficiency in subambient pressure ionization with nanoelectrospray. Anal. Chem. 82, 9344-9349 (2010)

10. Chen, L.C., Mandal, M.K., Hiraoka, K.: High pressure ( $>1$ atm) electrospray ionization mass spectrometry. J. Am. Soc. Mass Spectrom. 22, 539-544 (2011)

11. Bruins, A.P., Covey, T.R., Henion, J.D.: Ion spray interface for combined liquid chromatography/atmospheric pressure ionization mass spectrometry. Anal. Chem. 59, 2642-2646 (1987)

12. Takáts, Z., Wiseman, J.M., Gologan, B., Cooks, R.G.: Electrosonic spray ionization. A gentle technique for generating folded proteins and protein complexes in the gas phase and for studying ion-molecule reactions at atmospheric pressure. Anal. Chem. 76, 4050-4058 (2004)

13. Ikonomou, M.G., Kebarle, P.: A heated electrospray source for mass spectrometry of analytes from aqueous solutions. J. Am. Soc. Mass Spectrom. 5, 791-799 (1994)

14. Ikonomou, M. G., Blades, A. T., Kebarle, P. Electrospray mass spectrometry of methanol and water solutions suppression of electric discharge with $\mathrm{SF}_{6}$ gas. J. Am. Soc. Mass Spectrom. 2, 497-505 (1991)

15. Wilm, M.S., Mann, M.: Electrospray and Taylor-Cone theory, Dole's beam of macromolecules at last? Int. J. Mass. Spectrom. Ion Processes 136, 167-180 (1994)

16. Wilm, M., Mann, M.: Analytical properties of the nanoelectrospray ion source. Anal. Chem. 68, 1-8 (1996) 
17. López-Herrera, J.M., Barrero, A., Boucard, A., Loscertales, I.G., Márquez, M.: An experimental study of the electrospraying of water in air at atmospheric pressure. J. Am. Soc. Mass Spectrom. 15, 253-259 (2004)

18. Taylor, G.: Disintegration of water drops in an electric field. Proc. $R$. Soc. London, Ser. A 280, 383-397 (1964)

19. Smith, D.P.H.: The Electrohydrodynamic atomization of liquids. IEEE Trans. Ind. Appl IA-22, 527-535 (1986)

20. Paschen, F.: Ueber die zum Funkenübergang in Luft. Wasserstoff und Kohlensäure bei verschiedenen Drucken erforderliche Potentialdifferenz. Ann. Phys. 273, 69-96 (1889)

21. Wampler, F.M., Blades, A.T., Kebarle, P.: Negative ion electrospray mass spectrometry of nucleotides: ionization from water solution with SF6 discharge suppression. J. Am. Soc. Mass Spectrom. 4, 289-295 (1993)

22. Cloupeau, M., Prunet-Foch, B.: Electrohydrodynamic spraying functioning modes: a critical review. J. Aerosol Sci. 25, 1021-1036 (1994)

23. Tahallah, N., Pinkse, M., Maier, C.S., Heck, A.J.R.: The effect of the source pressure on the abundance of ions of noncovalent protein assemblies in an electrospray ionization orthogonal time-of-flight instrument. Rapid Commun. Mass Spectrom. 15, 596-601 (2001)

24. Schmidt, A., Bahr, U., Karas, M.: Influence of pressure in the first pumping stage on analyte desolvation and fragmentation in nano-ESI MS. Anal. Chem. 73, 6040-6046 (2001)

25. Van Berkel, W.J.H., Van Den Heuvel, R.H.H., Versluis, C., Heck, A.J. R.: Detection of intact megaDalton protein assemblies of vanillylalcohol oxidase by mass spectrometry. Protein Sci. 9, 435-439 (2000)
26. Kelly, R.T., Tolmachev, A.V., Page, J.S., Tang, K., Smith, R.D.: The ion funnel: theory, implementations, and applications. Mass Spectrom. Rev. 29, 294-312 (2010)

27. Kantrowitz, A., Grey, J.: A high intensity source for the molecular beam. Part I. Theoretical. Rev. Sci. Instrum. 22, 328 (1951)

28. Hirabayashi, A., Sakairi, M., Koizumi, H.: Sonic spray ionization method for atmospheric pressure ionization mass spectrometry. Anal. Chem. 66, 4557-4559 (1994)

29. Ohring, M. Vacuum science and technology. In: Materials Science of Thin Films (Second Edition). Academic Press: San Diego, 2002, pp. 57-93.

30. Lin, B., Sunner, J.: Ion transport by viscous gas flow through capillaries. J. Am. Soc. Mass Spectrom. 5, 873-885 (1994)

31. Nemes, P., Goyal, S., Vertes, A.: Conformational and noncovalent complexation changes in proteins during electrospray ionization. Anal. Chem. 80, 387-395 (2008)

32. Konermann, L., Douglas, D.J.: Unfolding of proteins monitored by electrospray ionization mass spectrometry: a comparison of positive and negative ion modes. J. Am. Soc. Mass Spectrom. 9, 1248-1254 (1998)

33. Schmidt, A., Karas, M.: The influence of electrostatic interactions on the detection of heme-globin complexes in ESI-MS. J. Am. Soc. Mass Spectrom. 12, 1092-1098 (2001)

34. Wu, C., Siems, W.F., Hill, H.H.: Secondary electrospray ionization ion mobility spectrometry/mass spectrometry of illicit drugs. Anal. Chem. 72, 396-403 (2000)

35. Fernandez de la Mora, J.: Ionization of vapor molecules by an electrospray cloud. Int. J. Mass. Spectrom 300, 182-193 (2011) 